\title{
Sleep Deprivation Impairs Cooperative Behavior Selectively: Evidence from Prisoner's and Chicken Dilemmas
}

This article was published in the following Dove Press journal: Nature and Science of Sleep

\author{
Yi Lin ${ }^{1} *$ \\ Ping $\mathrm{Hu}^{1,2, *}$ \\ Zifeng Mai ${ }^{1,2}$ \\ Tianxiang Jiang ${ }^{1,2}$ \\ Lei Mo ${ }^{1,3,4}$ \\ Ning $\mathrm{Ma}^{\mathrm{l}-4}$ \\ 'School of Psychology, ${ }^{2}$ Center for Sleep \\ Research, ${ }^{3}$ Center for Studies of \\ Psychological Application, ${ }^{4}$ Guangdong \\ Key Laboratory of Mental Health \& \\ Cognitive Science, South China Normal \\ University, Guangzhou 510631, People's \\ Republic of China \\ *These authors contributed equally to \\ this work
}

\begin{abstract}
Objective: The aim of our study was to investigate the influences of acute sleep deprivation on cooperation with two classical social dilemmas, the Prisoner's dilemma (PD) and the chicken dilemma (CD).

Methods: All participants $(\mathrm{N}=24)$ were required to come for the experiments twice; one time for normal sleep condition, the other time for sleep deprivation condition, with a counter-balanced sequence. In the following afternoon, participants completed the psychomotor vigilance task (PVT) and two social dilemmas tasks, as well as the Karolinska Sleepiness Scale (KSS), the Risk Orientation Questionnaire (ROQ) and the Positive and Negative Affect Schedule (PANAS).

Results: Our results demonstrated that sleep deprivation significantly impaired cooperative behaviors in the $\mathrm{CD}$ but not in the PD. In addition, this detrimental effect was not related with the alteration in the risk-seeking, objective alertness, subjective sleepiness, and mood.

Conclusion: The current findings revealed that sleep deprivation impairs cooperative behaviors under social dilemmas selectively. However, the underlying mechanism remains to further explore with neuroimaging studies and better ecological approach.
\end{abstract}

Keywords: sleep deprivation, cooperation, risk-seeking

\section{Introduction}

"No man is an island entire of itself; every man is a piece of the continent, a part of the main." Just as the poet John Donne said, cooperation, simply defined as two or more people working together toward a corporate aim, is inevitable owing to its fundamental role of social interaction. Doubtlessly, cooperation, a prerequisite of group, organization, and society, is essential not only for human evolution, but also for individual development, organizational management, and social progress. ${ }^{1}$

The social dilemma is one of the common approaches for investigating human cooperative behavior, mainly including two dilemma prototypes: the prisoner's dilemma (PD) and the chicken dilemma (CD, also known as the hawk-and-dove game). ${ }^{2,3}$ In both scenarios, two players choose to cooperate or defect, and each of them is paid according to mutual choice made by these two players. Considering individual player's payoffs, unilateral defection in the PD game benefits the most, followed by mutual cooperation, then mutual defection, and finally unilateral cooperation. In the CD game, unilateral defection benefits the most, followed by mutual cooperation, then unilateral cooperation, and finally mutual defection. More specifically, the PD game encourages people to defect with others, because mutual defection is a dominant-strategy equilibrium although 
mutual defection is worse for both players than mutual cooperation. ${ }^{4}$ However, there is no dominating strategy in the CD game. It forces people to make hard decisions between the balance of cooperation and defection. Defection in the CD is a high-risk decision situation with considerable outcome variance, which may cause the best or worst outcome. On the other hand, the risk level for choosing cooperation in the CD is relatively low, because the player may receive or lose intermediate pay-offs. Compared with PD, the mutual defection payoff in the $\mathrm{CD}$ is worse than the payoff for cooperation from just player. ${ }^{5}$ Therefore, the CD game may be more dynamic and ubiquitous than the PD game because players get immediate benefits from cooperation and costs of cooperation are shared. ${ }^{6}$

Cooperation is essential for social interaction, but people do not always cooperate with each other due to the universality and complexity of social dilemmas. Prior studies revealed that cooperation relies on reputation or potential future interactions, as well as propensity toward risk. $^{7,8}$ Recent studies applied two-person prisoner's games with gambles and found that risk-seeking had a positive correlation with cooperation, ${ }^{9,10}$ which is contradictory to earlier research suggesting no relationship between cooperation and risk preference. ${ }^{11}$ In addition, the relationship between risk-seeking and cooperation may be more influential in the $\mathrm{CD}$ than in the PD. De Heus and colleagues (2010) found a decreased rate of cooperative behavior in the $\mathrm{CD}$ caused by risk-seeking but not in the PD. ${ }^{5}$ Therefore, risk-taking preferences may play different roles in cooperative behavior with different social dilemmas.

Sleep loss has detrimental effects on a wide array of psychological functions including alertness, emotion, working memory, and reasoning. ${ }^{12,13}$ In the past decade, the effect of sleep loss on human social behavior has attracted increasing attention in the domain of sleep research. Although sleep loss significantly impairs an individual's performance, several studies consistently found that the social interaction or collaboration might be helpful to counter the adverse effect of sleep loss and partially improve individual's outcomes. ${ }^{14-16}$ In 2010, Anderson and Dickinson explored sleep deprivation and its effect on decision-making by using social economic games and observed lower trustworthiness and increased unfairness when people were sleep deprived. ${ }^{17} \mathrm{~A}$ recent study further demonstrated that chronic sleep loss significantly reduced people's trustworthiness and altruistic behavior. ${ }^{18}$

However, social relationships in daily life involve perceptions of others, such as trustworthiness and fairness, and also interaction with mutual interests and conflicts. Social dilemmas are the important approach to investigate cooperative behaviors based on the conflict of "individual needs and desires versus the benefit of the group." ${ }^{\prime 19}$ To date, no single study investigated the effect of acute sleep deprivation on cooperation with social dilemmas. In the current study, we applied two social dilemmas with different payoff matrixes (see Figure 1), in which the PD game leads to a win or nothing (zero gain) and the CD game leads to a win or loss (subtraction of money), to investigate whether sleep deprivation alters cooperative behaviors.

The other aim was to explore potentially influential factors underlying cooperative behaviors and sleep deprivation, including objective alertness, subjective sleepiness, mood, and risk-seeking. Numerous studies demonstrated that sleep loss strongly affected individual's alertness and sleepiness, ${ }^{20-26}$ as well as $\operatorname{mood}^{12}$ and risk-seeking. ${ }^{27-29}$ Those factors may also influence cooperative behavior due to insufficient resources to regulate cognitive and affective control after sleep loss. ${ }^{30}$ Based on these considerations,

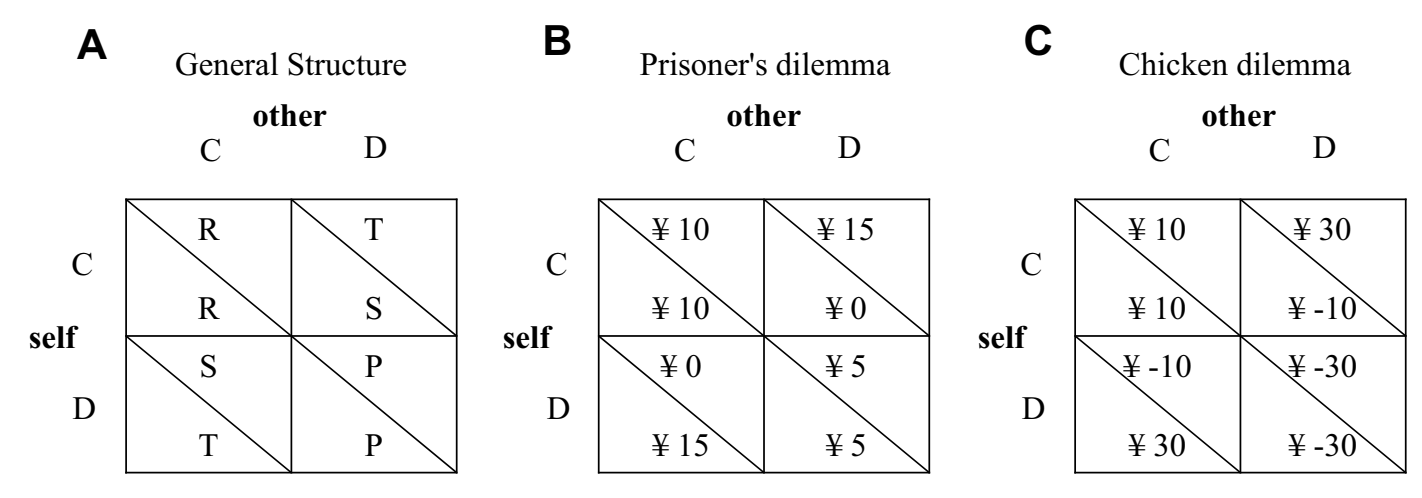

Figure I (A) Payoff matrix for general structure. (B) Payoff matrix for the prisoner's dilemma. (C) Payoff matrix for the chicken dilemma. Abbreviations: $C$, cooperation; $D$, detection; $P$, punishment; $R$, reward; S, sucker; T, temptation. 
we hypothesized that sleep deprivation might impair cooperative behaviors to different degrees in the $\mathrm{CD}$ and PD. The change of objective and subjective indicators (including objective alertness, subjective sleepiness, mood, and risk-seeking) may influence cooperative behaviors between normal sleep and sleep deprivation.

\section{Methods and Materials}

\section{Participants}

Twenty-four healthy adults $\left(\mathrm{M}_{\text {age }}=21.38 \pm 2.14\right.$ years old, 16 females) with normal body mass index $\left(19.97 \pm 1.86 \mathrm{~kg} / \mathrm{m}^{2}\right)$ and normal or corrected-to-normal vision participated in this study, which was conducted in the Center for Sleep Research at the South China Normal University. All study procedures were approved by the Ethical Committee of the South China Normal University, and were conducted in accordance with the Declaration of Helsinki. All participants provided written informed consent and were financially compensated for their time.

All the participants were nonsmokers. They had no acute or chronic physical or psychological conditions, as established by interviews, clinical history, questionnaires, and physical examinations. Participants had habitual 6.5-8 hrs sleep duration and habitual awake times between 06:00 am and 09:00 am, as assessed by sleep diary and actigraphy (Actiwatch Spectrum, Philips) within 2 weeks before the in-laboratory experiment. Individuals with sleep disorders were excluded by the sleep adaptation night of laboratory polysomnography (PSG) and oximetry measurements (Grael HD PSG, Compumedics). Participants had no transmeridian travel or shift work (in any pattern, including evening or night shifts, early morning shifts, and rotating shifts) or irregular sleep-wake routines in 60 days prior to the in-lab experiment. No caffeine, alcohol, or medications were permitted 3 days before and during the in-laboratory study.

\section{Experimental Procedures}

All participants were required to come to the Center for Sleep Research twice, one time for normal sleep condition, the other for sleep deprivation condition. The condition sequence was counter-balanced. If the experiment started with normal sleep, the sleep deprivation session was carried out after 1 week to minimize the practice effects of behavioral tasks. If the session of sleep deprivation started first, the normal sleep session was after at least 1 month to ensure participants fully recovered from the effect of one-night acute sleep deprivation, and to minimize the practice effects of behavioral tasks as well.

Participants were continuously monitored in a semiisolated living area and only had contact with research staff during the stay in the lab. They were given meals at regular, pre-specified hours (7:30 am for breakfast, 12:00 pm for lunch, and 18:00 pm for dinner). Meals contained no caffeinated products. During their free time, participants were permitted to read books and magazines and converse with the study monitors. They were not allowed to do any physically demanding tasks. Participants were not allowed to use light-emitting electronic equipment, such as smartphones or tablets, during the whole experiment. They were only allowed to use desktop computers during behavioral tests on the study time schedule.

For the in-laboratory protocol (Figure 2), during the normal sleep condition, participants arrived at the laboratory in the evening around 19:00 pm and had around $8 \mathrm{hrs}$ of time in bed (normally starting from 23:00 pm, the duration of normal sleep was $7.99 \pm 0.28 \mathrm{hrs}$ according to the PSG data) for baseline sleep. During the sleep deprivation condition, participants did not sleep for the whole night under monitoring by the research staff. In addition, all the participants were required to have around $8 \mathrm{hrs}$ of time in bed as adapting sleep in the laboratory the night before the experiment (normally starting from 23:00 $\mathrm{pm}$, the duration of normal sleep was $8.07 \pm 0.32 \mathrm{hrs}$ according to PSG data) to familiarize participants with

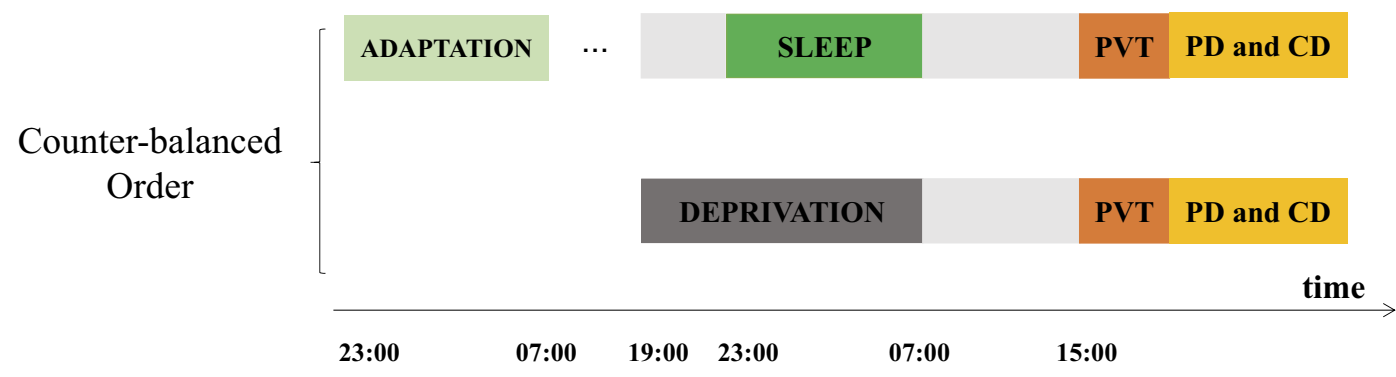

Figure 2 Experimental protocol of normal sleep and sleep deprivation conditions. 
the experimental environment and avoid potential factors influencing their sleep. In the afternoon following the normal sleep or sleep deprivation night, participants filled in the Positive and Negative Affect Schedule (PANAS), Risk Orientation Questionnaire (ROQ), and Karolinska Sleepiness Scale (KSS) scales and then performed a psychomotor vigilance task followed by the $\mathrm{PD}$ and CD. The task sequence of PD and CD was counterbalanced between conditions (Normal sleep/Sleep deprivation) and participants.

\section{Experimental Paradigms and Instruments Prisoner's Dilemma (PD) and the Chicken Dilemma (CD)}

In both the $\mathrm{PD}$ and $\mathrm{CD}$ games, participants were told that they would be paid each round based on the decisions made by themselves and their partners, who were 30 different people that only contacted them via the computer (but their partners were actually fictive and randomly selected by computer, and the participants did not know that their partners were fictive). The participants had to make a choice between A (cooperation) or B (defection), but the words "cooperation" and "defection" were never used. Participants were asked to make their own choice without being informed about the partner's choice. To avoid potential influence of the prior response on the next decision, there was no feedback about the outcome during the experiment.

The instructions for both games with the payoff matrix (see Figure 2) were always shown on the screen during the game. ${ }^{5}$ To make sure the participants understood the games, we also explained the implications of the payoff matrix for both the PD and CD to the participants before the experiment. In the PD game, the B option always leads to a better outcome for oneself than the A option. However, if both of the players choose B, both will get worse outcomes than if both chose $\mathrm{A}$. In the $\mathrm{CD}$ game, one may get the best possible gain with option $\mathrm{B}$ (if the other chooses A) but may also get the worst loss (if the other chooses B). Option A always leads to an intermediate outcome, either a gain or a loss.

\section{Psychomotor Vigilance Test (PVT)}

Under both normal sleep and sleep deprivation conditions, participants were administered a 10 mins version of the PVT before the cooperation tasks. ${ }^{31}$ This sustained attention task is based on visual reaction time, which is highly sensitive to the circadian and homeostatic influences on cognitive decrements due to sleep loss. ${ }^{20-23}$ Participants were asked to focus their attention on a red, rectangular box subtending $2 \times 1.3$ degrees of visual angle in the middle of a black screen and monitor that space for the appearance of a millisecond counter, which appeared at random intervals ranging from 2-10 s. They were instructed to stop the counter as quickly as possible with a button press, after which they would be able to view their reaction time. Participants were also instructed to avoid anticipating the stimuli so as not to register "false starts," or responses when no stimulus was present on the screen.

\section{Subjective Sleepiness, Mood and Risk-Seeking Preference}

Subjective sleepiness was assessed by the KSS. ${ }^{32}$ The KSS is a 9-point, verbally anchored scale ranging from 1 (extremely alert) to 9 (extremely sleepy).

The PANAS is a 5-point scale with two subscales (positive and negative affect) was applied to measure participants' affective state. It was developed by Watson and colleagues and adapted to the Chinese population in $2008{ }^{33,34}$ We used the Chinese version, which includes 20 items with 10 positive affect and 10 negative affect items.

We also used the ROQ to assess individuals' riskseeking level under normal sleep and sleep deprivation conditions. ${ }^{35}$ The ROQ is a 7-point scale containing 12 items to evaluate how different people deal with risky situations and assess their attitude toward risk decision.

\section{Results}

\section{Differences of Objective Alertness and Subjective Ratings of Sleepiness, Mood, and Risk-Seeking Preference Between Normal Sleep and Sleep Deprivation}

The number of PVT lapses (reaction time $>500 \mathrm{~ms}$ ) and the reciprocal of mean reaction time are two common indicators to assess alertness attention after sleep deprivation. The increased number of PVT lapses (paired $t$-test: $\mathrm{t}=-4.71, p<0.001$, Cohen's $\mathrm{d}=1.34)$ and the decreased reciprocal of mean reaction time (paired $t$-test: $\mathrm{t}=6.48$, $p<0.001$, Cohen's $\mathrm{d}=-0.98$ ) indicated impaired participant alertness due to sleep insufficiency (Table 1).

We used paired t-tests to investigate whether sleep deprivation altered the ratings of subjective sleepiness, as well as negative affect (Table 1). As expected, compared to the normal sleep condition, the sleepiness rating was marginally increased $(\mathrm{t}=-1.890, p=0.071$, Cohen's $\mathrm{d}=-0.39)$ after 
Table I Objective Measurements and Subjective Ratings During Normal Sleep and Sleep Deprivation

\begin{tabular}{|l|l|l|l|}
\hline & $\begin{array}{l}\text { Normal } \\
\text { Sleep }\end{array}$ & $\begin{array}{l}\text { Sleep } \\
\text { Deprivation }\end{array}$ & $p$ \\
\hline $\begin{array}{l}\text { PVT } \\
\text { I/mean RT }\end{array}$ & $3.24 \pm 0.34$ & $2.12 \pm 0.74$ & $<0.001$ \\
Number of lapses & $3.46 \pm 2.78$ & $17.00 \pm 15.18$ & $<0.00 \mathrm{I}$ \\
\hline $\begin{array}{l}\text { Cooperation Rate } \\
\text { (\%) }\end{array}$ & & & \\
PD & $28.69 \pm 29.54$ & $30.49 \pm 31.51$ & 0.992 \\
CD & $80.19 \pm 26.75$ & $58.01 \pm 37.76$ & 0.007 \\
\hline Subjective Rating & & & \\
Sleepiness & $4.78 \pm 1.21$ & $6.42 \pm 1.64$ & 0.061 \\
PANAS (P) & $28.92 \pm 7.64$ & $25.58 \pm 9.44$ & 0.020 \\
PANAS (N) & $14.25 \pm 6.04$ & $18.17 \pm 6.79$ & 0.004 \\
ROQ & $44.70 \pm 3.21$ & $47.00 \pm 6.59$ & 0.057 \\
\hline
\end{tabular}

Abbreviations: $C D$, chicken dilemma; PANAS, Positive and Negative Affect Schedule; PD, prisoner's dilemma; PVT, Psychomotor Vigilance Test; ROQ, Risk Orientation Questionnaire; RT, response time.

sleep deprivation. Analysis of PANAS scores showed that positive affect (paired $t$-test: $\mathrm{t}=2.51, p=0.02$, Cohen's $\mathrm{d}=$ 0.51 ) and negative affect (paired $t$-test: $\mathrm{t}=-3.22, p=0.004$, Cohen's $d=-0.66$ ) were significantly reduced due to sleep loss.

Paired t-tests were used to assess risk seeking by comparing ROQ ratings between normal sleep and sleep deprivation. The result showed a marginal difference $(\mathrm{t}=-2.00$, $p=0.057$, Cohen's $\mathrm{d}=-0.409$ ) between normal sleep and sleep deprivation conditions.

\section{Cooperation Performance Affecting by Sleep Deprivation}

Repeated measures analysis of variance revealed that a significant main effect between different cooperative tasks $\left(\mathrm{F}=20.1, p<0.001\right.$, partial $\left.\eta^{2}=44.0 \%\right)$. Participants chose to cooperate at a higher rate in the CD task than in the PD task, regardless of sleep state. The main effect of sleep state was also significant $\left(\mathrm{F}=4.93, p=0.037\right.$, partial $\eta^{2}=$ $17.7 \%)$, and there was a significant interaction effect $(\mathrm{F}=$ $6.99, p=0.014$, partial $\eta^{2}=23.3 \%$ ). A post hoc comparison revealed that the rate of cooperation decreased after sleep deprivation in the $\mathrm{CD}$ tasks $(\mathrm{t}=3.438, p=0.007)$ but not in the PD tasks $(\mathrm{t}=-0.279, p=0.992$, Table 2, Figure 3).

\section{Correlations Analysis Between Cooperation and Objective/Subjective Indicators}

To clarify the processes underlying different cooperative tasks after normal sleep and sleep deprivation, we analyzed the relationship between the change of cooperative tasks and objective alertness (PVT performance), as well as with subjective ratings (subjective sleepiness, positive/ negative affect, and risk preference). However, the analysis did not reveal any significant correlation (Table 2).

\section{Discussion}

The present study investigated whether sleep deprivation affects cooperative behavior in two social dilemmas, the PD and CD. In line with previous studies, the CD had a higher level of cooperation than the PD. Moreover, sleep deprivation decreased the cooperation rate in the CD task but not the PD task, and the impairment of cooperation in $\mathrm{CD}$ by sleep deprivation was not associated with differences of objective alertness, subjective fatigue, mood, or risk-seeking between normal sleep and sleep deprivation.

In the two classic social dilemmas, an individual makes the decision based on his/her own will and the other's choice. Generally speaking, in the PD task, people may have an optimal strategy, which is defection regardless of the other's choice, to ensure stable payoff for himself/

Table 2 Correlations Between Cooperation Changes and Indicators' Changes

\begin{tabular}{|c|c|c|c|c|c|c|c|c|}
\hline & & & \multicolumn{6}{|c|}{ Indicators (Normal Sleep vs Sleep Deprivation) } \\
\hline & & & $\begin{array}{l}\text { Number of } \\
\text { Lapses }\end{array}$ & $\begin{array}{l}\text { I/Mean } \\
\text { RT }\end{array}$ & Sleepiness & $\begin{array}{l}\text { PANAS } \\
\text { (P) }\end{array}$ & $\begin{array}{l}\text { PANAS } \\
\text { (N) }\end{array}$ & $\begin{array}{l}\text { ROQ } \\
\text { Ratings }\end{array}$ \\
\hline \multirow{4}{*}{$\begin{array}{l}\text { Cooperation (normal sleep vs sleep } \\
\text { deprivation) }\end{array}$} & \multirow[t]{2}{*}{ PD } & $r$ & -0.008 & -0.067 & 0.291 & 0.028 & -0.014 & 0.326 \\
\hline & & $p$ & 0.972 & 0.755 & 0.167 & 0.895 & 0.587 & 0.119 \\
\hline & \multirow[t]{2}{*}{$C D$} & $r$ & 0.057 & 0.260 & 0.373 & 0.075 & -0.062 & 0.002 \\
\hline & & $p$ & 0.793 & 0.221 & 0.073 & 0.732 & 0.770 & 0.992 \\
\hline
\end{tabular}

Abbreviations: CD, chicken dilemma; PANAS, Positive and Negative Affect Schedule; PD, prisoner's dilemma; ROQ, Risk Orientation Questionnaire; RT, response time. 


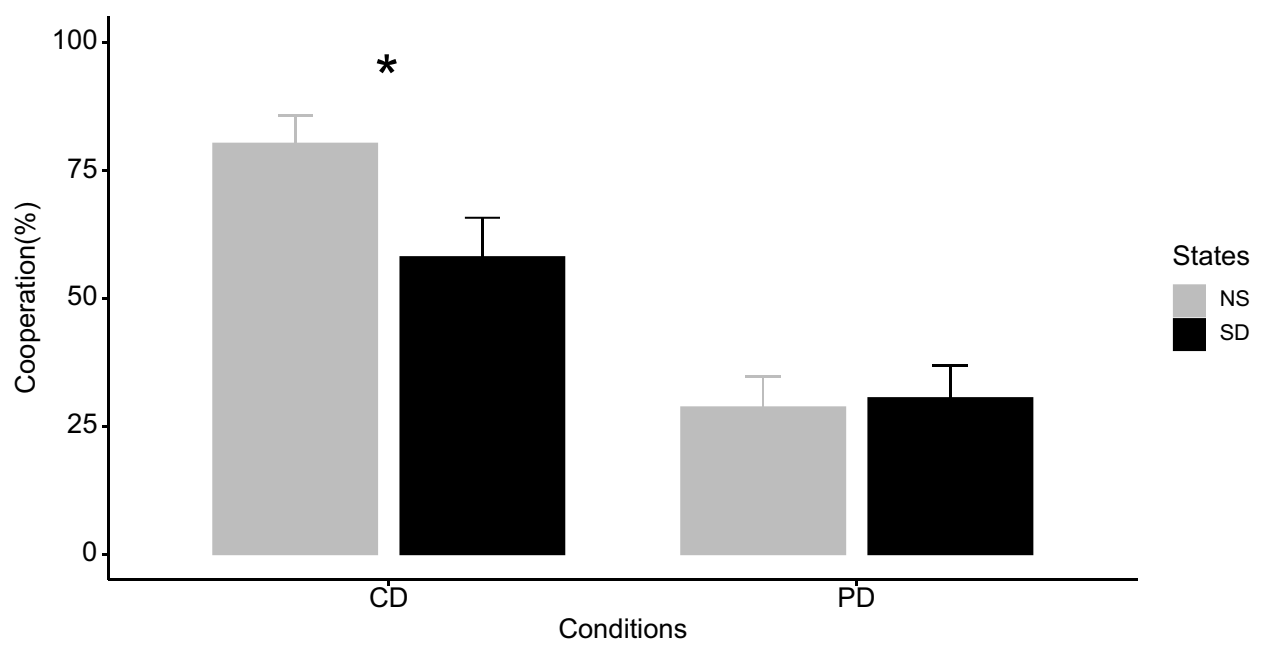

Figure 3 Cooperation rates for CD and PD under normal sleep and sleep deprivation condition.

Note: ${ }^{*} p=0.007$.

Abbreviations: $C D$, chicken dilemma; PD, prisoner's dilemma.

herself. However, in the CD task, there is no such strategy to keep stable payoff if one only considers his/her own interests and ignore the other's choice. ${ }^{6}$ In this way, it is reasonable for people to choose cooperation in the $\mathrm{CD}$ task more than in the PD task, which is in line with the current results. In addition, the rate of cooperation did not change much in the PD task but decreased significantly in the CD task after sleep deprivation. It seems that sleep deprivation did not alter the rate of defection much in the PD game, but it decreased cooperative behavior in the CD game. In addition, the $\mathrm{CD}$ game involves greater risk (more is at stake and more can be won or lost), compared to the PD game that is either a win or nothing. That is, it seems that sleep deprivation has a greater effect when the stakes are higher.

The influence of sleep deprivation on human cooperation may be related to several factors. First, decreased cooperative behavior may result from enhanced risk-seeking. ${ }^{5,9,10,36}$ In the current study, we tested the level of risk-seeking with the ROQ and found only marginal differences between the normal sleep and sleep deprivation conditions. Some studies demonstrated that acute sleep-deprived individuals are more sensitive to gain and less sensitive to loss, compared with the well-rested condition, ${ }^{37,38}$ which might be explained by elevated risk propensity and attenuated risk aversion after sleep loss. ${ }^{27-29}$ However, other evidence indicated that risky decision-making did not affect by sleep loss robustly. ${ }^{39-42}$ These inconsistent findings may indicate that risk-seeking is not strongly impaired by sleep deprivation and helps to explain why the change in risk-seeking did not correlate with differences in cooperation rates in the PD and CD tasks between normal sleep and sleep deprivation. Second, numerous studies have indicated that sleep deprivation decreases objective alertness and dramatically enhances subjective sleepiness. ${ }^{20-26}$ Low alertness and fatigue make it difficult to carefully process information, which may influence cooperative behavior. However, decreased alertness (the number lapses and reciprocal of mean reaction time of PVT) and increased sleepiness (KSS scores) were not significantly correlated with the change in the rates of cooperation in PD and CD tasks. Moreover, we also found that the differences in the two tasks between normal sleep and sleep deprivation were not affected by mood, although both negative and positive affect were significantly changed due to sleep insufficiency. This indicated that differences of objective and subjective indicators might not influence cooperation in the dilemmas after sleep deprivation.

\section{Limitations and Future Directions}

The present study demonstrated that sleep deprivation selectively impairs cooperative behaviors in social dilemmas, but the results should be considered in the context of several limitations. First, the sample size was relatively small. Individual differences in how people respond to sleep deprivation may partially explain why the present study did not uncover the influential factors (e.g., risk-seeking, objective alertness, subjective sleepiness, and mood), which may be related with the change in cooperation after sleep loss. Additionally, the time of day effect on objective performance and subjective ratings may also need more attention. Although 
objective PVT performance significantly declined after sleep deprivation, subjective sleepiness was only marginally decreased. This may be related with the time when the measurements were taken. All the behavioral tasks and rating were performed at $15 \mathrm{pm}$ in the afternoon. According to the twoprocess model of sleep regulation, ${ }^{43,44}$ individuals may perform worse in the afternoon even with enough sleep, as well as provide higher sleepiness ratings. This may explain why the KSS results were only marginally different after sleep deprivation. Correspondingly, this may also influence the correlation between sleepiness and cooperative behavior. Future studies need to recruit larger samples and consider different time points to further clarify how sleep deprivation affects cooperation.

Secondly, most of the variables we used to examine potential influences on cooperative behavior after sleep deprivation were subjective evaluations, such as questionnaires about risk-seeking, positive and negative mood, and sleepiness. Subjective measurements may not sufficiently reflect the status or performance of individuals after sleep deprivation. Specially, the ROQ applied in the current study was mainly used for measuring the stable propensity of risk, which may be not suitable to test state effects. This could explain the marginal state difference of risk-seeking between the normal sleep and sleep deprivation conditions. It is better to investigate impaired effects of risk-taking propensity after sleep deprivation by both self-report and behavioral measurement ${ }^{45}$ and to consider assessing differences in other types of behavioral performance (e.g., trust propensity) to explore potential influences on cooperative behavior between normal sleep and sleep deprivation.

Thirdly, sensitivity of gain and loss is crucial for promoting cooperation in social dilemmas. Several studies have verified that sleep deprivation impairs human incentive processing, and neuroimaging evidence suggests that sleep loss causes diminished medial prefrontal cortex responses to gain outcomes and increased anterior insula responses to loss outcomes. ${ }^{12,46}$ Future studies may apply functional neuroimaging techniques to investigate if the change in cooperative behavior after sleep deprivation correlated with sensitivity of gain and loss.

Last but not least, the findings of the current study need further verification by actual face-to-face interactions in realworld contexts. We explicitly controlled potential confounders, such as face-to-face interactions and feedback after single trials, to try to reduce the effects of other factors. However, social interactions are very common and clearly interesting components in real-world life. Future study is needed to explore the effects of sleep loss on social behavior with better ecological approaches.

\section{Conclusion}

The present study suggested that sleep deprivation selectively impairs cooperative behavior, demonstrated by the decreased rate of cooperation in the CD but not in the PD after sleep loss. In addition, the differences of cooperation between normal sleep and sleep deprivation in the CD game were not correlated with changes in objective alertness, subjective sleepiness, negative affect, or risk preference. Unfortunately, the current study did not uncover the mechanism by which sleep deprivation affects people's cooperation, but it may help rule out several possible factors (e.g., riskseeking level, alertness, sleepiness, and mood). Future investigations should recruit larger sample with better ecological approaches to verify the current findings with other potential mediators affecting cooperation (e.g., trustworthiness, sensitivity of gain and loss) and use neuroimaging techniques to further explore human cooperative behaviors after sleep loss.

\section{Acknowledgments}

This research was supported by the National Natural Science Foundation of China (31500906), Natural Science Foundation of Guangdong Province - General Program (2019A1515012182) and Guangdong Provincial Youth Innovation Talent Project (2017WQNCX021).

\section{Author Contributions}

All authors contributed to design of the study, data analysis, drafting or revising the article, gave final approval of the version to be published, and agree to be accountable for all aspects of the work.

\section{Disclosure}

The authors report no conflicts of interest in this work.

\section{References}

1. The cooperative human. Nat Hum Behav. 2018;2:427-428. doi:10. 1038/s41562-018-0389-1

2. Axelrod R, Hamilton WD. The evolution of cooperation. Science. 1981;211(4489):1390-1396. doi:10.1126/science.7466396

3. Smith JM, Price GR. The logic of animal conflict. Nature. 1973;246 (5427):15. doi:10.1038/246015a 0

4. Lipman BL. Cooperation among egoists in prisoners' dilemma and chicken games. Public Choice. 1986;51(3):315-331. doi:10.1007/ bf00128880

5. De Heus P, Hoogervorst N, Van Dijk E. Framing prisoners and chickens: valence effects in the prisoner's dilemma and the chicken game. $J$ Exp Soc Psychol. 2010;46(5):736-742. doi:10.1016/j.jesp.2010.04. 013 
6. Kümmerli R, Colliard C, Fiechter N, Petitpierre B, Russier F, Keller L. Human cooperation in social dilemmas: comparing the Snowdrift game with the prisoner's dilemma. Proc Biol Sci. 2007;274(1628):2965-2970. doi:10.1098/rspb.2007.0793

7. Schlaeper A. The emergence and selection of reputation systems that drive cooperative behavior. Proc Biol Sci. 2018;285(1886):20181508. doi:10.1098/rspb.2018.1508

8. Güney Ş, Newell BR. Fairness overrides reputation: the importance of fairness considerations in altruistic cooperation. Front Hum Neurosci. 2013;7:252. doi:10.3389/fnhum.2013.00252

9. Raub W, Snijders C. Gains, losses, and cooperation in social dilemmas and collective action: the effects of risk preferences. $J$ Math Sociol. 1997;22(3):263-302. doi:10.1080/0022250X.1997.9990204

10. Van Assen MALM, Snijders CCP. The effect of nonlinear utility on behaviour in repeated prisoner's dilemmas. Rationality Soc. 2010;22 (3):301-332. doi:10.1177/1043463110374499

11. Dolbear FT, Lave LB. Risk orientation as a predictor in the prisoner's dilemma. J Conflict Resolut. 1966;10(4):506-515. doi:10.1177/ 002200276601000409

12. Krause AJ, Simon EB, Mander BA, et al. The sleep-deprived human brain. Nat Rev Neurosci. 2017;18(7):404. doi:10.1038/nrn.2017.55

13. Lim J, Dinges DF. A meta-analysis of the impact of short-term sleep deprivation on cognitive variables. Psychol Bull. 2010;136(3):375. doi:10.1037/a0018883

14. Baranski JV, Thompson MM, Lichacz FMJ, et al. Effects of sleep loss on team decision making: motivational loss or motivational gain? Hum Factors. 2007;49(4):646-660. doi:10.1518/001872007X215728

15. Holding BC, Sundelin T, Lekander M, Axelsson J. Sleep deprivation and its effects on communication during individual and collaborative tasks. Sci Rep. 2019;9(3131):1-8. doi:10.1038/s41598-019-39271-6

16. Pilcher JJ, Vander Wood MA, O'Connell KL. The effects of extended work under sleep deprivation conditions on team-based performance. Ergonomics. 2011;54(7):587-596. doi:10.1080/00140139.2011.592 599

17. Anderson C, Dickinson DL. Bargaining and trust: the effects of 36-h total sleep deprivation on socially interactive decisions. J Sleep Res. 2010;19(1):54-63. doi:10.1111/j.1365-2869.2009.00767.x

18. Dickinson DL, McElroy T. Sleep restriction and circadian effects on social decisions. Eur Economic Rev. 2017;97:57-71. doi:10.1016/j. euroecorev.2017.05.002

19. Dawes RM. Social dilemmas. Annu Rev Psychol. 1980;31:169-193. doi:10.1146/annurev.ps.31.020180.001125

20. Doran SM, Van Dongen HPA, Dinges DF. Sustained attention performance during sleep deprivation: evidence of state instability. Arch Ital Biol. 2001;139(3):253-267. doi:10.4449/aib.v139i3.503

21. Dorrian J, Rogers NL, Dinges DF. Psychomotor vigilance performance: Neurocognitive assay sensitive to sleep loss. In: Kushida CA editor. Sleep Deprivation: Clinical Issues, Pharmacology, and Sleep Loss Effects. Boca Raton, Florida: CRC Press; 2004:67-98.

22. Lim J, Dinges DF. Sleep deprivation and vigilant attention. Ann N Y Acad Sci. 2008;1129(1):305-322. doi:10.1196/annals.1417.002

23. Basner M, Dinges DF. Maximizing sensitivity of the psychomotor vigilance test (PVT) to sleep loss. Sleep. 2011;34(5):581-591. doi:10.1093/sleep/34.5.581

24. Philip P, Taillard J, Sagaspe P, et al. Age, performance and sleep deprivation. J Sleep Res. 2004;13(2):105-110. doi:10.1111/j.13652869.2004.00399.x

25. Chee MW, Chuah LY, Venkatraman V, Chan WY, Philip P, Dinges DF. Functional imaging of working memory following normal sleep and after 24 and $35 \mathrm{~h}$ of sleep deprivation: correlations of fronto-parietal activation with performance. Neuroimage. 2006;31 (1):419-428. doi:10.1016/j.neuroimage.2005.12.001

26. Slama H, Chylinski DO, Deliens G, Leproult R, Schmitz R, Peigneux P. Sleep deprivation triggers cognitive control impairments in task-goal switching. Sleep. 2017;41(2):zsx200. doi:10.1093/sleep/ zsx 200
27. Venkatraman V, Huettel SA, Chuah LY, Payne JW, Chee MW. Sleep deprivation biases the neural mechanisms underlying economic preferences. $J$ Neurosci. 2011;31(10):3712-3718. doi:10.1523/JNEURO SCI.4407-10.2011

28. Bayard S, Langenier MC, Dauvilliers Y. Decision-making, reward-seeking behaviors and dopamine agonist therapy in restless legs syndrome. Sleep. 2013;36(10):1501-1507. doi:10.5665/sleep. 3044

29. Womack SD, Hook JN, Reyna SH, Ramos M. Sleep loss and risk-taking behavior: a review of the literature. Behav Sleep Med. 2013;11(5):343-359. doi:10.1080/15402002.2012.703628

30. Schmidt RE, Harvey AG, Van der Linden M. Cognitive and affective control in insomnia. Front Psychol. 2011;2:349. doi:10.3389/ fpsyg.2011.00349

31. Dinges DF, Pack F, Williams K, et al. Cumulative sleepiness, mood disturbance, and psychomotor vigilance performance decrements during a week of sleep restricted to $4-5$ hours per night. Sleep. 1997;20 (4):267-277. doi:10.1093/sleep/20.4.267

32. Akerstedt T, Gillberg M. Subjective and objective sleepiness in the active individual. Int J Neurosci. 1990;52(1-2):29-37. doi:10.3109/ 00207459008994241

33. Watson D, Clark LA, Tellegen A. Development and validation of brief measures of positive and negative affect: the PANAS scales. J Pers Soc Psychol. 1988;54(6):1063. doi:10.1037/0022-3514.54.6.1063

34. Qiu L, Zheng X, Wang YF. Revision of the positive affect and negative affect scale. Chin J Appl Psychol. 2008;14(3):249-254.

35. Rohrmann B. Risk Attitude Scales: Concepts and Questionnaires. Melbourne: University of Melbourne; 2002:12.

36. Glöckner A, Hilbig BE. Risk is relative: risk aversion yields cooperation rather than defection in cooperation-friendly environments. Psychon Bull Rev. 2012;19(3):546-553. doi:10.3758/s13423-0120224-z

37. Mckenna BS, Dickinson DL, Orff HJ, Drummond SP. The effects of one night of sleep deprivation on known-risk and ambiguous-risk decisions. J Sleep Res. 2007;16(3):245-252. doi:10.1111/j.13652869.2007.00591.x

38. Venkatraman V, Chuah YL, Huettel SA, Chee MW. Sleep deprivation elevates expectation of gains and attenuates response to losses following risky decisions. Sleep. 2007;30(5):603-609. doi:10.1093/ sleep/30.5.603

39. Maric A, Montvai E, Werth E, et al. Insufficient sleep: enhanced risk-seeking relates to low local sleep intensity. Ann Neurol. 2017;82:409-418. doi:10.1002/ana.25023

40. Menz MM, Buchel C, Peters J. Sleep deprivation is associated with attenuated parametric valuation and control signals in the midbrain during value-based decision making. J Neurosci. 2012;32:6937-6946. doi:10.1523/JNEUROSCI.3553-11.2012

41. Mullette-Gillman OA, Kurnianingsih YA, Liu JC. Sleep deprivation alters choice strategy without altering uncertainty or loss aversion preferences. Front Neurosci. 2015;9:352. doi:10.3389/fnins.2015.00 352

42. Sundelin T, Bayard F, Schwarz J, et al. Framing effect, probability distortion, and gambling tendency without feedback are resistant to two nights of experimental sleep restriction. Sci Rep. 2019;9:8554. doi:10.1038/s41598-019-44237-9

43. Borbély AA. A two process model of sleep regulation. Hum Neurobio. 1982;1(3):195-204.

44. Borbély AA, Daan S, Wirz-Justice A, et al. The two-process model of sleep regulation: a reappraisal. J Sleep Res. 2016;25(2):131-143. doi: $10.1111 /$ jsr. 12371

45. Killgore WD. Effects of sleep deprivation and morningness-eveningness traits on risk-taking. Psychol Rep. 2007;100:613-626. doi:10.2466/ pr0.100.2.613-626

46. Killgore WD, Balkin TJ, Wesensten NJ. Impaired decision making following 49 h of sleep deprivation. J Sleep Res. 2006;15(1):7-13. doi:10.1111/j.1365-2869.2006.00487.x 


\section{Publish your work in this journal}

Nature and Science of Sleep is an international, peer-reviewed, open access journal covering all aspects of sleep science and sleep medicine, including the neurophysiology and functions of sleep, the genetics of sleep, sleep and society, biological rhythms, dreaming, sleep disorders and therapy, and strategies to optimize healthy sleep.
The manuscript management system is completely online and includes a very quick and fair peer-review system, which is all easy to use. Visit http://www.dovepress.com/testimonials.php to read real quotes from published authors. 